\title{
Diagnosis, prognostic significance, and characteristics of new-onset right bundle-branch block in patients with acute myocardial infarction: Protocol for a systematic review and meta-analysis
}

\author{
Juntao Wang ${ }^{\# *}$, Jialu Zhu* , Hongxing Luo, Chunling Kong, Cong Zhang, Yingjie Chu
}

\begin{abstract}
Wang J, Zhu J, Luo H, et al. Diagnosis, prognostic significance, and characteristics of new-onset right bundle-branch block in patients with acute myocardial infarction: Protocol for a systematic review and meta-analysis. Curr Res Cardiol 2017;4(3):40-44.

BACKGROUND: Many studies have shown that patients with acute Myocardial Infarction (AMI) and Bundle Branch Block (BBB) may have a worse prognosis than patients with only acute myocardial infarction. Current guidelines recommend reperfusion therapy in patients with ST. segment elevation AMI or left bundle branch block AMI. Curiously, the right bundle-branch block (RBBB) is not listed as an indication for reperfusion therapy. However, some studies did not find a significant prognostic value of RBBB in acute myocardial infarction. Furthermore, distinction between old and new bundle-branch block is seldom made in previously studies. Our study is to investigate the incidence, clinical characteristics, diagnosis, and prognostic significance of new-onset RBBB in acute myocardial infarction.
\end{abstract}

METHODS AND ANALYSIS: A systematic review of studies evaluating

\begin{abstract}
the prognosis of right and left bundle-branch block in acute myocardial infarction will be undertaken. PubMed, EMBASE, Ovid, Cochrane Library and Web of Science will be searched electronically. Reference lists of related reviews, included studies and key journals will be manual-searched to identify further studies for inclusion. Two reviewers will independently complete publication type. Title, abstract and full-text will be screened to determine study eligibility according to the Patient-Intervention-ControlOutcomes (PICO) criteria. Data in the final included studies will be extracted. Quality assessment of included studies will be evaluated using the Newcastle-Ottawa Quality Assessment Scale. Stata 22.0 will process the data.
\end{abstract}

ETHICS AND DISSEMINATION: As data in this systematic review and meta-analysis is based on published articles, ethics approval is not required. Dissemination will be undertaken through publication in peer-reviewed journals, and the lead author's master dissertation.

CONCLUSION: Findings may provide with valid and precise risk estimates of new-onset RBBB in AMI for clinicians and other decision-makers.

Key Words: Acute myocardial infarction; Bundle branch block; Prognosis
$\mathrm{M}$ any studies have shown that patients with AMI and bundlebranch block (BBB) may have a worse prognosis than patients with only AMI. Yet investigators of these studies did not compare the effects of new-RBBB versus old-RBBB (1). A recent systematic review (2) showed that patients with RBBB and AMI were at more than 2-fold higher risk of all-cause mortality at 30 days of follow up compared to those with no BBB. Furthermore, for patients with myocardial infarction, several other studies have reported positive associations between RBBB and all-cause mortality (3-5), whereas others have reported no association $(6,7)$.

Department of Cardiology, Zhengzhou University People's Hospital, No.7 Weiwu Road, Zhengzhou, Henan, P.R. China

\# The authors have equally contributed to the study.

Correspondence: Dr. Juntao Wang, Department of Cardiology, Zhengzhou University People's Hospital, No.7 Weiwu Road, Zhengzhou 450000, Henan, P.R. China, Telephone+8615639029039, e-mail cardiacresearch-wang@foxmail.com

Received: Jul 27, 2017, Accepted: Sep 04, 2017; Published: Sep 06, 2017

This open-access article is distributed under the terms of the Creative Commons Attribution Non-Commercial License (CC BY-NC) work is properly cited and the reuse is restricted to noncommercial purposes. For commercial reuse, contact reprints@pulsus.com 
Considering the anatomy and vascular supply of the conduction system $(8,9)$, RBBB is usually the manifestation of large infarctions that are often accompanied by heart failure, complete AV block, arrhythmias, and a high mortality rate (10-20). The classification of RBBB according to timing, duration, and association with fascicular block is of clinical importance $(17,18,21)$. However, studies of RBBB in AMI took place in the prethrombolytic era claimed that, thrombolytic treatment limits infarct size $(22,23)$, improves ventricular morphology and function (24), and decreases mortality (25-28). Moreover, studies have connected the reversibility of conduction disturbances with coronary reperfusion $(29,30)$, which suggests that reperfusion therapy can prevent the appearance or limit the duration of bundle-branch blocks. Thus, it is probable that the current wide use of reperfusion therapy may have changed the overall incidence and significance of RBBB in AMI and therefore it is reasonable to reanalyze its meaning in the reperfusion therapy era.

Moreover, the American Heart Association and European Society of Cardiology in their guidelines consider AMI patients with new-onset LBBB a high-risk group and recommend for their treatment early reperfusion therapy with percutaneous coronary intervention or fibrinolytic therapy (31,32). Yet both organizations acknowledge that it is difficult to diagnose ST elevation MI in the setting of LBBB and ascertain whether the LBBB is old or new, considering that oftentimes no prior electrocardiogram is available for comparison. However, the RBBB is not listed as an indication for reperfusion therapy.

Accordingly, we are conducting a systematic review and meta-analysis to assess the diagnosis, prognostic value of new RBBB in patients with AMI, in terms of risk for short- and long-time all-cause mortality, cardiac death, malignant arrhythmia, risk of heart failure, and so on.

\section{METHODS AND ANALYSIS}

This protocol was developed from the recommendations in the statement on Preferred Reporting Items for Systematic Review and Meta-Analyses Protocol (33), and is registered in PROSPERO website, (PROSPERO Registration Number: CRD42017070425). We will conduct and report this review according to the PRISMA statement (34).

\section{Eligibility criteria}

A study will be included for review if it is a full-text, original article which reports about the prognosis developed from AMI+new-RBBB, such as mortality, heart failure, complete AV block, arrhythmias and so on. We will apply the following inclusion and exclusion criteria:

\section{Inclusion criteria}

- Articles that report on prognosis in the population of individuals with AMI.

- Articles reporting new-RBBB and old-RBBB.

- Articles in English.

\section{Exclusion criteria}

- Studies are not original study.

- Studies did not report the outcomes after the AMI.

- Studies in which we are failed to obtain accurate data.

- Articles not written in English will be excluded. system cause.

\section{Search strategy}

On June 13, 2017, we searched the following 5 databases with similar combined items as follows:

PubMed 373: (Acute myocardial infarction [Title/Abstract] OR AMI [Title/Abstract] OR Acute heart infarction [Title/Abstract]) AND (("Bundle-Branch Block"[Mesh]) OR (((bundle branch block [Title/Abstract]) OR bundle-branch block [Title/Abstract]) OR BBB [Title/Abstract])) AND (("Prognosis"[Mesh]) OR ("Survival Analysis"[Mesh]) OR ("Mortality"[Mesh]) OR $\quad((((($ prognosis [Title/Abstract]) OR survival analysis [Title/Abstract]) OR mortality [Title/Abstract]) OR death [Title/Abstract]) OR outcome [Title/Abstract]) OR Follow-up [Title/Abstract]))

\section{Cochrane Library 66:}

\#1 Acute myocardial infarction or AMI or Acute heart infarction: ti,ab,kw (Word variations have been searched) 11242

\#2 MeSH descriptor: [Bundle-Branch Block] explode all trees 137

\#3 bundle branch block or bundle-branch block or BBB:ti,ab,kw (Word variations have been searched) 563

\#4 \#2 or \#3 563

\#5 MeSH descriptor: [Prognosis] explode all trees 138441

\#6 MeSH descriptor: [Survival Analysis] explode all trees 18933

\#7 MeSH descriptor: [Mortality] explode all trees 13098

\#8 prognosis or survival analysis or mortality or death or outcome or Follow-up: ti,ab,kw (Word variations have been searched) 397004

\#9 \#5 or \#6 or \#7 or \#8 400951

$\# 10$ \#1 and \#4 and \#9 66

EMBASE 765: 'acute heart infarction'/exp OR 'acute myocardial infarction':ab,ti OR 'ami':ab,ti OR 'acute heart infarction':ab,ti AND 
('heart bundle branch block'/exp OR 'bundle branch block':ab,ti OR 'bundle-branch block':ab,ti OR 'bbb':ab,ti) AND ('prognosis'/exp OR 'survival analysis'/exp OR 'mortality'/exp OR 'follow up'/exp OR 'prognosis':ab,ti OR 'survival analysis':ab,ti OR 'mortality':ab,ti OR 'death':ab,ti OR 'outcome':ab,ti OR 'follow-up':ab,ti)

\section{Ovid (MEDLINE) 383:}

1. (Acute myocardial infarction or AMI or Acute heart infarction).ab,kf,ti.

2. exp Bundle-Branch Block/

3. (bundle branch block or bundle-branch block or BBB).ab,kf,ti

4. 2 or 3

5. exp Prognosis/

6. exp Survival Analysis/

7. exp Mortality/

8. exp Follow-Up Studies/

9. (prognosis or survival analysis or mortality or death or outcome or Follow-up).ab,kf,ti.

10. 5 or 6 or 7 or 8 or 9

11. 1 and 4 and 10

Web of Science 381: TS= (Acute myocardial infarction OR AMI OR Acute heart infarction) AND TS= (bundle branch block OR bundlebranch block OR BBB) AND TS= (prognosis OR survival analysis OR mortality OR death OR outcome OR Follow-up).

\section{Study records}

Results of searched literature will be saved and the titles and abstracts of identified studies will be downloaded to NoteExpress Management software (v3.2.0.6941). Two reviewers will screen the items independently follow the eligibility criteria online (https://www.covidence.org/). After the screening of titles and abstracts, full-text will be downloaded of the remaining records. Then final set of records for inclusion will be obtained after further screening according to inclusion and exclusion criteria described in subsequent paragraphs. The reason for the exclusion will be logged in a table at the full-text stage. A third reviewer will be delegated to the discrepancies through discussion at the full-text stage. We will use a PRISMA flow diagram (35) to record the process of information acquisition during the review phases.
Data will be independently extracted by two researchers. We extracted the following items according to a uniform data extraction form:

- Author

- Year of publication

- Study design

- Study period

- Population characteristics (e.g., age, sex)

- Follow-up duration

- $\quad$ Sample size \& new-RBBB number

- Location

- Patient involvement in outcomes selection

Outcome

Primary outcome: The incidence of cardiac death.

Secondary outcomes: major adverse cardiovascular events and allcause mortality., malignant, risk of heart failure.

1. Arrhythmia

- Rapid supraventricular arrhythmia (atrial flutter and atrial fibrillation, supraventricular tachycardia)

- Bradycardia (sinoatrial/atrioventricular block above two degrees)

- Malignant ventricular arrhythmia (ventricular tachycardia, ventricular flutter and ventricular fibrillation)

2. Heart failure

3. Cardiogenic shock

4. All-cause mortality

Risk of bias (quality) assessment

We will assess the quality of included studies using the risk of bias tool of Newcastle-Ottawa Quality Assessment. A table will be made to record the risk of bias and quality scores. Funnel plots will be made to explore the effect size of small studies, and Egger's test will be used to test the symmetry.

\section{Statistical analysis}

Estimates for the prognosis after AMI+new-RBBB will be pooled into a meta-analysis and displayed with 95\% confidence intervals.

A random effect will be used to obtain an overall summary estimate of the incidence across studies by pooling the study specific estimates.

Data items 
Statistical heterogeneity across studies will be quantified by calculating I2 and assessed by the Cochrane Q test. If the value of I2 $>50 \%$, there is substantial heterogeneity. We will conduct a subgroup analysis to investigate the possible reasons of heterogeneity if heterogeneity is statistically significant according to the following variables: type of new-RBBB, age, country, combined disease, and so on. Furthermore, we will perform a sensitivity analysis to find out if only high-quality studies were considered how the results would change. Publication bias across studies or selective reporting bias will be assessed through the symmetry of the funnel plot. Data will be analyzed using Stata V14.0 for Windows.

\section{DISCUSSION}

This systematic review and meta-analysis will define the incidence data for new-RBBB in the patients after AMI. To describe the prognosis of AMI+new-RBBB, such as all-cause mortality, arrhythmia, etc. Compared with the old-RBBB, indicating the relationship between the new-RBBB and the prognosis, providing a reference for clinicians and evidence for decision maker whether to change the relevant guidelines.

Due to the language barrier, only English articles will be included in our study. This may be another limitation of this study, for which we may lose relevant data from non-English spoken areas and may cause publication bias to some extent.

\section{REFERENCES}

1. Bhalli MA, Khan MQ, Samore NA, et al. Frequency and clinical outcome in conduction defects in acute myocardial infarction. J Ayub Med Coll Abbottabad. 2009;21(3):32-7.

2. Hazem A, Sharma S, Sharma A, et al. Is right bundle branch block associated with poor outcomes in the setting of an acute coronary syndrome? A systematic review and meta-analysis. Circ Cardiovasc Qual Outcomes. 2014;7(Suppl 1): A309.

3. Kleemann $\mathrm{T}$, Juenger $\mathrm{C}$, Gitt $\mathrm{AK}$, et al. Incidence and clinical impact of right bundle branch block in patients with acute myocardial infarction: ST elevation myocardial infarction versus non-ST elevation myocardial infarction. Am Heart J. 2008;156(2): 256-61.

4. Widimsky P, Rohác F, Stásek J, et al. Primary angioplasty in acute myocardial infarction with right bundle branch block: should new onset right bundle branch block be added to future guidelines as an indication for reperfusion therapy? Eur Heart J. 2012;33:86-95.

5. Wong CK, Stewart RAH, Gao W, et al. Prognostic differences between different types of bundle branch block during the early phase of acute myocardial infarction: insights from the Hirulog and Early Reperfusion or Occlusion (HERO)-2 trial. Eur Heart J.

\section{STRENGTHS}

This systematic review will provide evidence about the incidence and prognostic significance of new-onset RBBB in patients with acute myocardial infarction.

\section{LIMTATIONS}

Limitations include an acknowledged language bias, as only studies published in English will be included. Furthermore, grey literature and ongoing research will also not be included in our study and this may cause publication bias to some extent.

\section{CONCLUSION}

A subgroup analysis, such as new-onset permanent or transient RBBB, thrombolysis or percutaneous coronary intervention, will make it possible to distinguish different prognosis in specific populations and screen the populations for optimum therapy.

\section{DECLARATION OF CONFLICT OF INTEREST} None.

\section{TRIAL REGISTRATION NUMBER}

\section{CRD42017070425.}

2006;27(1):21-8.

6. Archbold RA, Sayer JW, Ray S, et al. Frequency and prognostic implications of conduction defects in acute myocardial infarction since the introduction of thrombolytic therapy. Eur Heart J. 1998;19(6):893-8.

7. Juarez-Herrera U, Jerjes-Sanchez C. Risk factors, therapeutic approaches, and in-hospital outcomes in Mexicans with ST-elevation acute myocardial infarction: The RENASICA II multicenter registry. Clin Cardiol. 2013;36(5):241-8.

8. Mullins CB, Atkins JM. Prognoses and management of venticular conduction blocks in acute myocardial infarction. Mod Concepts Cardiovasc Dis. 1976;45(10):129-33.

9. Roos JC, Dunning AJ. Bundle branch block in acute myocardial infarction. Eur J Cardiol. 1978;6(6):403-24.

10. Julian DG, Valentine PA, Miller GG. Disturbances of rate, rhythm and conduction in acute myocardial infarction: a prospective study of 100 consecutive unselected patients with the aid of electrocardiographic monitoring. Am J Med. 1964;37:915-27.

11. Col JJ, Weinberg SL. The incidence and mortality of intraventricular conduction defects in acute myocardial infarction. Am J Cardiol. 1972;29(3):344-50. 
12. Rizzon P, Biase DM, Baissus C. Intraventricular conduction defects in acute myocardial infarction. Br Heart J. 1974;36(7): 660-8.

13. Mullins CB, Atkins JM. Prognoses and management of venticular conduction blocks in acute myocardial infarction. Mod Concepts Cardiovasc Dis. 1976;45(10): 129-33.

14. Medrano GA, De Micheli A. Disorders of intraventricular conduction in acute myocardial infarct. Arch Inst Cardiol Mex. $1980 ; 50(3): 341-53$

15. Roos JC, Dunning AJ. Bundle branch block in acute myocardial infarction. Eur J Cardiol. 1978;6(6):403-24.

16. Klein RC, Vera Z, Mason DT. Intraventricular conduction defects in acute myocardial infarction: incidence, prognosis, and therapy. Am Heart J. 1984;108:1007-13.

17. Hindman MC, Wagner GS, Ja-Ro M, et al. The clinical significance of bundle branch block complicating acute myocardial infarction. 1 . Clinical characteristics, hospital mortality, and one-year follow-up. Circulation. 1978;58(4):679-88.

18. Lie KI, Wellens HJ, Schuilenburg RM, et al., Factors influencing prognosis of bundle branch block complicating acute antero-septal infarction. The value of his bundle recordings. Circulation. 1974;50(5):935-41.

19. Lie KI, Liem KL, Schuilenburg RM, et al. Early identification of patients developing late in-hospital ventricular fibrillation after discharge from the coronary care unit. A 5 1/2-year retrospective and prospective study of 1,897 patients. Am J Cardiol. 1978;41(4):674-7.

20. Hauer RN, Lie KI, Liem KL, et al. Long-term prognosis in patients with bundle branch block complicating acute anteroseptal infarction. Am J Cardiol. 1982;49(7):1581-5.

21. Ricou F, Nicod P, Gilpin E, et al. Influence of right bundle branch block on short- and long-term survival after acute anterior myocardial infarction. J Am Coll Cardiol. 1991;17(4):858-63.

22. Kloner RA, Ellis SG, Lange R, et al. Studies of experimental coronary artery reperfusion. Effects on infarct size, myocardial function, biochemistry, ultrastructure and microvascular damage. Circulation. 1983;68:I8-15.

23. Braunwald E. The path to myocardial salvage by thrombolytic therapy. Circulation. 1987;76:II2-7.

24. White HD, Norris RM, Brown MA, et al. Effect of intravenous streptokinase on left ventricular function and early survival after acute myocardial infarction. N Engl J Med. 1987;317(14):850-5.
25. Yusuf S, Sleight $\mathrm{P}$, Held $\mathrm{P}$, et al. Routine medical management of acute myocardial infarction. Lessons from overviews of recent randomized controlled trials. Circulation. 1990;82(3Suppl):II11734.

26. Grines CL, DeMaria AN. Optimal utilization of thrombolytic therapy for acute myocardial infarction: Concepts and controversies. J Am Coll Cardiol. 1990;16(1):223-31.

27. Nicod P, Zimmermann M, Scherrer U. The challenge of further reducing cardiac mortality in the thrombolytic era. Circulation. 1993;87(2):640-2.

28. Indications for fibrinolytic therapy in suspected acute myocardial infarction: Collaborative overview of early mortality and major morbidity results from all randomised trials of more than 1000 patients. Fibrinolytic Therapy Trialists' (FTT) Collaborative Group. Lancet. 1994;343(8893):311-22.

29. Roth A, Miller HI, Glick A, et al. Rapid resolution of new right bundle branch block in acute anterior myocardial infarction patients after thrombolytic therapy. Pacing Clin Electrophysiol.1993;16:13-8.

30. Wiseman A, Ohman EM, Wharton JM. Transient reversal of bifascicular block during acute myocardial infarction with reperfusion therapy: A word of caution. Am Heart J. 1989;117(6):1381-3.

31. Emergency department: Rapid identification and treatment of patients with acute myocardial infarction. National Heart Attack Alert Program Coordinating Committee, 60 Minutes to Treatment Working Group. Ann Emerg Med. 1994;23(2):311-29.

32. Guyatt GH, Oxman AD, Vist G, et al. GRADE guidelines: 4. Rating the quality of evidence-study limitations (risk of bias). J Clin Epidemiol. 2011;64(4):407-15.

33. Shamseer L, Moher D, Clarke M, et al. Preferred reporting items for systematic review and meta-analysis protocols (PRISMA-P) 2015: elaboration and explanation. BMJ. 2015;349:g7647.

34. Hutton B, Salanti G, Caldwell DM, et al., The PRISMA extension statement for reporting of systematic reviews incorporating network meta-analyses of health care interventions: checklist and explanations. Ann Intern Med. 2015;162(11):777-84.

35. Stovold E, Beecher D, Foxlee R, et al. Study flow diagrams in Cochrane systematic review updates: an adapted PRISMA flow diagram. Syst Rev. 2014;3:54. 